\title{
Guideline for the evaluation of prescription appropriateness
}

Jing Bian ${ }^{1 \#}$, Quanzhi Li ${ }^{1 \#}$, Jing Li ${ }^{1 \#}$, Nan Yang ${ }^{2,3}$, Wei Zhang ${ }^{1}$, Dan Mei ${ }^{4}$, Mingkang Zhong ${ }^{5}$, Liyan Miao ${ }^{6}$, Xiaoyang $\mathrm{Lu}^{7}$, Ling Jiang ${ }^{8}$, Guang Du ${ }^{9}$, Shen Gao ${ }^{10}$, Xuehua Jiang ${ }^{11}$, Jin Lu ${ }^{12}$, Bing Yan ${ }^{13}$, Fancui Kong ${ }^{13}$, Yaolong Chen ${ }^{2,3}$, Jiancun Zhen ${ }^{1}$; represent the guideline expert group from the Pharmaceutical Affairs Commission of the Chinese Hospital Association

${ }^{1}$ Department of Pharmacy, Beijing Jishuitan Hospital, Beijing, China; ${ }^{2}$ Evidence-Based Medicine Center, School of Basic Medical Sciences, Lanzhou University, Lanzhou, China; ${ }^{3}$ Chinese GRADE Center, Lanzhou University, Lanzhou, China; ${ }^{4}$ Department of Pharmacy, Peking Union Medical College Hospital, Beijing, China; ${ }^{5}$ Department of Pharmacy, Huashan Hospital Affiliated to Fudan University, Shanghai, China; ${ }^{6}$ Department of Pharmacy, the First Affiliated Hospital of Suzhou University, Suzhou, China; ${ }^{7}$ Department of Pharmacy, the First Affiliated Hospital of Zhejiang University School of Medicine, Hangzhou, China; ${ }^{8}$ Department of Pharmacy, Anhui Provincial Hospital, Hefei, China; ${ }^{9}$ Department of Pharmacy, Tongji Hospital, Tongji Medical College, Huazhong University of Science and Technology, Wuhan, China; ${ }^{10}$ Department of Pharmacy, Changhai Hospital, Shanghai, China; ${ }^{11}$ Department of Pharmacy, West China Research Center of Clinical Pharmacy of Sichuan University, Chengdu, China; ${ }^{12}$ Department of Pharmacy, China-Japan Friendship Hospital, Beijing, China; ${ }^{13}$ Department of Pharmaceutical Affairs, Beijing Hospitals Authority, Beijing, China

Contributions: (I) Conception and design: All authors; (II) Administrative support: J Zhen, Y Chen; (III) Provision of study materials or patients: J Bian, Q Li, J Li; (IV) Collection and assembly of data: J Bian, Q Li, J Li, N Yang; (V) Data analysis and interpretation: J Bian, Q Li, J Li, N Yang; (VI) Manuscript writing: All authors; (VII) Final approval of manuscript: All authors.

"These authors contributed equally to this work.

Correspondence to: Yaolong Chen. No. 199, Donggang Road (West), Chengguan District, Lanzhou, China. Email: chenyaolong@vip.163.com; Jiancun Zhen. 31 Xinjiekou E Rd, Xicheng District, Beijing, China. Email: zhenjiancun@vip.163.com.

\begin{abstract}
Evaluation of prescriptions is a necessary process of evaluating the appropriateness of clinical drug usage, discovering existing problems, and formulating solutions. There are challenges for professionals within hospital medical departments and for clinicians and pharmacists who have clinical questions relating to inappropriate or abnormal prescriptions as identified by the electronic evaluation system of prescription. Medications are usually used correctly according to the drug instructions or guidelines. At present, there are no relevant domestic or international guidelines, or principles or standards for identifying inappropriate or abnormal prescriptions. To develop the guideline for evaluation of prescriptions appropriateness in clinical practice, the Pharmaceutical Affairs Commission of the Chinese Hospital Association formed the guideline working group consisting of multidisciplinary experts. The guideline working group summarized clinical questions in the evaluation of prescriptions, searched for supporting evidence, and reached a consensus for recommendations. The guideline contains 6 recommendations for evaluating prescription appropriateness, and the general principle of these recommendations is that clinicians should provide drug instructions, guidelines, or moderate evidence supporting the prescription, and the evaluators will then judge the prescription to be either appropriate or irrational. The recommendations resolve common clinical questions, using supporting examples, explanations and a flow chart. The evaluation of prescription appropriateness could be made more systematic and transparent based on this guideline's conclusions.
\end{abstract}

Keywords: Prescription comment; inappropriate prescription; prescription error; guideline

Submitted Nov 16, 2020. Accepted for publication May 16, 2021.

doi: $10.21037 /$ atm-20-7502

View this article at: https://dx.doi.org/10.21037/atm-20-7502 


\section{Background}

The evaluation of prescriptions is the assessment of the appropriateness of clinical drug usage (drug indications, drug selection, route of administration, dosage, drug interactions, contraindications, etc.) according to relevant laws and technical specifications. By evaluation of prescriptions, existing or potential problems can be identified, and interventions formulated and implemented to promote the appropriateness of clinical drug usage (1). As the "Management Practices of Hospital Prescription Comment (Trial)" issued by the former Chinese Ministry of Health in 2010 states, irrational prescription can be divided into nonstandard prescriptions, inappropriate prescriptions, and abnormal prescriptions (1). Existing criteria concerning prescription appropriateness include the Beers Criteria, which is the standard tool used to characterize a potentially inappropriate prescriptions in the older adult population. However, these criteria only focus on the appropriateness of several prescriptions in specific populations. The evaluation of prescriptions must solve questions about the appropriateness of every prescription within the entire population. It is thus necessary to establish a process or procedure to guide hospital medical management department professionals, clinicians, and pharmacists to search for the best evidence for confirming a prescription's appropriateness. Hence, the Pharmaceutical Affairs Commission of the Chinese Hospital Association initiated this guideline for evaluating prescription appropriateness. The guideline aims to support the identification of inappropriate and abnormal prescriptions according to evidence-based methods.

We present the following article in accordance with the RIGHT (Reporting Items for Practice Guidelines in Healthcare) reporting checklist (available at https://dx.doi. org/10.21037/atm-20-7502).

\section{Methods}

\section{Composition of the guideline working group}

The guideline was launched by the Pharmaceutical Affairs Commission of the Chinese Hospital Association on February 22, 2019. A multidisciplinary working group, including experts from pharmacy, clinical medicine, evidence-based medicine, hospital management, law, and pharmaceutical economics was established to develop these guidelines. All members of the guideline working group declared that they had no conflicts of interest associated with the creation of the guideline.

\section{Methods and processes of development}

The guideline was developed with consideration to the World Health Organization Handbook for Guideline Development released in 2014 (2). The guideline conformed to the Regulations on Prescription Management and the Management Practices of Hospital Prescription Comment $(1,3)$ issued by the former Chinese Ministry of Health.

\section{Aims and use of the guideline}

The guideline's users are hospital medical management department professionals, clinicians, and pharmacists. There are some prescriptions that the electronic evaluation system of prescription cannot judge correctly. This guideline aims to help users solve clinical questions relating to the evaluation of such prescriptions.

\section{Selection and determination of the clinical questions}

A week in 2018 was chosen at random, and the information of 474,263 outpatient and emergency prescriptions from 22 hospitals in Beijing was sourced. All prescriptions were first evaluated by the electronic evaluation system of prescription, which contains the label information of commonly used medicines from different manufacturers and guideline information from authoritative associations, after which 5,648 prescriptions were identified as irrational. The working group then decided on 27 clinical questions by reviewing these prescriptions. The guideline working group had 2 rounds of voting for each recommendation using a slightly modified version of the Delphi method. Reaching a consensus required a vote of $75 \%$. Finally, based on the vote results and the comments, the guideline working group chose 6 common clinical questions and raised example analyses and related explanations for each question (shown in Appendix 1).

\section{Search and selection of evidence}

Focusing on the examples related to recommendations, we 
searched the following web sources and other guidelinerelated websites for relevant clinical practice guidelines: UpToDate, DynaMed, PubMed, EmBase, China Biology Medicine disc (CBM disc), China National Knowledge Infrastructure (CNKI), Wanfang Med Online, the National Institute for Health and Care Excellence (NICE), the National Guideline Clearinghouse (NGC), the Scottish Intercollegiate Guidelines Network (SIGN), the World Health Organization (WHO), Guidelines International Network (GIN), and the International Practice Guideline Registry Platform; for systematic reviews (SRs) and meta-analyses, we searched PubMed Cochrane Library Epistemonikos, CBM disc, Wanfang Med Online, and CNKI; for original studies, including randomized controlled trials (RCTs), cohort studies, case-control studies, case series, epidemiological investigations, and others, we searched UpToDate, DynaMed, PubMed, the CBM disc, Wanfang Med Online, and CNKI; for relevant notices, standards, and regulations, we searched other sites, such as those of the National Health Commission and the National Medical Products Administration (NMPA). According to the $5 \mathrm{~S}$ model of evidence-based medicine, guidelines, SRs, RCTs, observational studies, and other forms of evidence were selected in turn (4). All searches were carried out on October 31, 2019.

\section{Evaluation and grading of evidence}

The methodological quality of included guidelines was assessed by using AGREE II (5), while of the risk of evidence bias was evaluated using the following tools: (I) A Measurement Tool to Assess Systematic Reviews (AMSTAR) (6) for included SRs and meta-analyses, (II) the risk of bias (RoB) tool recommended by the Cochrane handbook for included RCTs (7), (III) Quality Assessment of Diagnostic Accuracy Studies (QUADAS-2) for included diagnostic accuracy studies (8), and (IV) the Newcastle-Ottawa Scale (NOS) for included observational studies (9). The evaluation was completed by 2 researchers independently, and all disagreements were resolved through discussion with a third researcher. The qualities of evidence were graded using the Grading of Recommendations Assessment, Development and Evaluation approach (GRADE) (10). The quality level of each document was classified as "high", "moderate", "low" or "very low".

\section{Formation of recommendations}

According to the Evidence to Decision (EtD) frameworks (11), the guideline working group formulated 6 recommendations determined by the following queries: (I) What is the level of certainty in local and external evidence? (II) What is the risk of irrational prescriptions? (III) Is the recommendation compliant with relevant policies and regulations? (IV) Is the recommendation feasible? In addition, the guideline also provided explanations and example analyses for each recommendation. The guideline working group conducted a survey for the recommendations of 24 experts from 21 hospitals in China from October 19 to 27, 2019, and collected 227 feedback suggestions. A face-to-face discussion and consensus meeting were held in Beijing on November 5, 2019, and the guideline's manuscript was finalized on December 31, 2019.

\section{Update of the guideline}

The recommendations in the guideline will be updated in 3 to 5 years as new evidence and processes emerge (12).

\section{Results}

\section{Clinical question 1}

Instructions of the same drug from different manufacturers are inconsistent for the applicable/contraindicated population. Should the prescription be judged as irrational if a clinician prescribes one drug according to the directions of one manufacturer that does not conform to the directions of another manufacturer?

\section{Recommendation}

The clinician should provide drug instructions for the prescription, explain the differences between the instructions from one drug manufacturer and the instructions of the same drug from the other manufacturer, discuss the possible differences of therapeutic effect, and further determine whether the prescription is appropriate based on the above information. It is suggested that the prescription comment system should promptly add and update the drug instructions of all manufacturers of the same drug. 


\section{Explanation}

In theory, drug instructions are approved by the NMPA and comply with national laws and regulations. However, in the process of drug production, different raw materials, technologies, and procedures may lead to differences in the usage and dosage of the same drug produced by different manufacturers. In general, the differences do not have significant impacts on a physician prescribing medication or treating a condition, but in some cases there may be significant differences between one manufacturer's drug instructions and those of another, which has the potential to impact a patient's health. In the event of such problems, the guideline suggests that the prescribing physician should review the instructions of this drug from different manufacturers to determine whether the prescription should be modified. In addition, the prescription comment system should collect as many drug instructions as possible from different manufacturers of the same drug to provide as much information to prescribing clinicians as is available.

\section{Clinical question 2}

If two different diseases have a similar pathogenesis, can a drug used to treat one disease be used to treat the other similar yet separate disease?

\section{Recommendation}

Clinicians should provide evidence for the efficacy and safety of the prescribed drug to treat another disease. If there is a high or moderate level of evidence supporting the treatment of the disease with a similar pathogenesis, the prescription can be considered appropriate; otherwise, it should be judged as irrational.

\section{Explanation}

Evidence of efficacy and safety should be provided for the off-label use of any medication. According to the $5 \mathrm{~S}$ model of evidence-based medicine (5), the lower the level of reference evidence is, the less rational the prescription is.

\section{Clinical question 3}

If the frequency or dose of a prescription exceeds the recommended amount; if the diagnosis of the prescription not included in the indications of the drug instructions; or if the diagnosis of the prescription is included in the contraindications of the drug, but the domestic guidelines nonetheless recommend the drug, how should the appropriateness of the prescription be evaluated?

\section{Recommendation}

Clinicians should evaluate the relevant domestic guidelines when prescribing. A prescription may be deemed appropriate if the guideline is issued by the appropriate authority (a national medical and health administration, association, or society).

\section{Explanation}

Guidelines issued by authorities with access to rigorous methodological guidance and expert review are generally of higher quality.

\section{Clinical question 4}

Can a prescription be considered appropriate if the indication, frequency, or dosage does not comply with the drug instructions and is not recommended by domestic guidelines or guidelines for special populations (e.g., overweight, diabetic, renal hypofunction patients) but is consistent with international guidelines or guidelines for normal populations?

\section{Recommendation}

Clinicians should search for evidence of the efficacy and safety of this drug within the domestic population or special populations, analyze whether it has been widely used in clinical practice, evaluate whether it is consistent with national or special conditions, elaborate on the reasons for it being prescribed, and examine relevant research evidence to judge whether the prescription is appropriate.

\section{Explanation}

International guidelines and guidelines for normal populations generally lack supporting evidence on the drug's usage in domestic and special populations, so clinicians need to comprehensively elaborate on the necessity of prescribing 
this drug.

\section{Clinical question 5}

If the indication, frequency, or dose of the prescription does not comply with the drug instructions, and the recommendation opinions of relevant guidelines are inconsistent, how should the appropriateness of the prescription be judged?

\section{Recommendation}

The prescribing clinician should first evaluate the quality of the relevant guidelines. Prescriptions can be judged as appropriate if they are based on higher quality guidelines.

\section{Explanation}

Guidelines of higher quality are formulated using more rigorous methods, and thus the recommendations are more reliable.

\section{Clinical question 6}

How should the appropriateness of the prescription be evaluated if the indication, frequency, or dose of the prescription does not comply with the drug instructions and if there are no relevant recommendations in domestic or international guidelines?

\section{Recommendation}

Clinicians should provide evidence for the efficacy and safety of the drug in the treatment of a diagnosed disease, evaluate the quality of the evidence, and grade the evidence using GRADE, elaborate the reasons for prescribing the drug, and compile relevant research reports. On this basis it can be determined if the prescription is appropriate.

\section{Explanation}

If there are no recommendations or guidelines it is necessary to search for effectiveness and safety studies then grade the evidence using GRADE. If there is a high or moderate level of evidence to support the indication, frequency, or dose of the prescription, the prescription can be considered appropriate; otherwise, it should be considered irrational.

\section{Discussion}

This guideline was developed following the guideline definition from the Institute of Medicine (IOM) with reference to the World Health Organization Handbook for Guideline Development. At the beginning of development of the guideline, a Delphi vote was used to determine 6 out of 27 clinical questions integrated from 5,648 prescriptions, which will contribute to the implementation of the guideline into clinical practice.

To be certain all experts formulated their recommendations objectively, experts' declarations of conflicts of interest were made clear. The group of experts formulated 6 recommendations in this guideline based on local and external research evidence and with consideration to multiple criteria in the $\mathrm{EtD}$ model. In addition, the guideline also provides explanations and example analyses for each recommendation. A comprehensive search strategy was used to identify as much relevant research as possible for the example analyses. We also used the GRADE approach to rate the quality of evidence. The strengths of the guideline are the systematic, practical, and evidence-based approach used in the development of the guideline. Moreover, a problematic prescription determination flow chart, shown in Figure 1, was developed.

\section{Limitations}

This guideline acknowledges its limitations. Recommendations could not be formulated for some clinical questions, as a consensus could not be reached. For example, it was not agreed upon how a prescription should be evaluated if it is contraindicated by the specific stage of a diagnosis in the prescription is a contraindication to a drug in the prescription. The recommendations of this guideline require a high level of evidence retrieval and evaluation by guideline users that may hinder the clinical application of the guideline.

\section{Conclusions}

Our guideline working group formulated 6 recommendations to resolve clinical questions relating to the evaluation of prescription appropriateness. We hope this guideline can facilitate the more systematic and transparent evaluation of irrational prescriptions. 


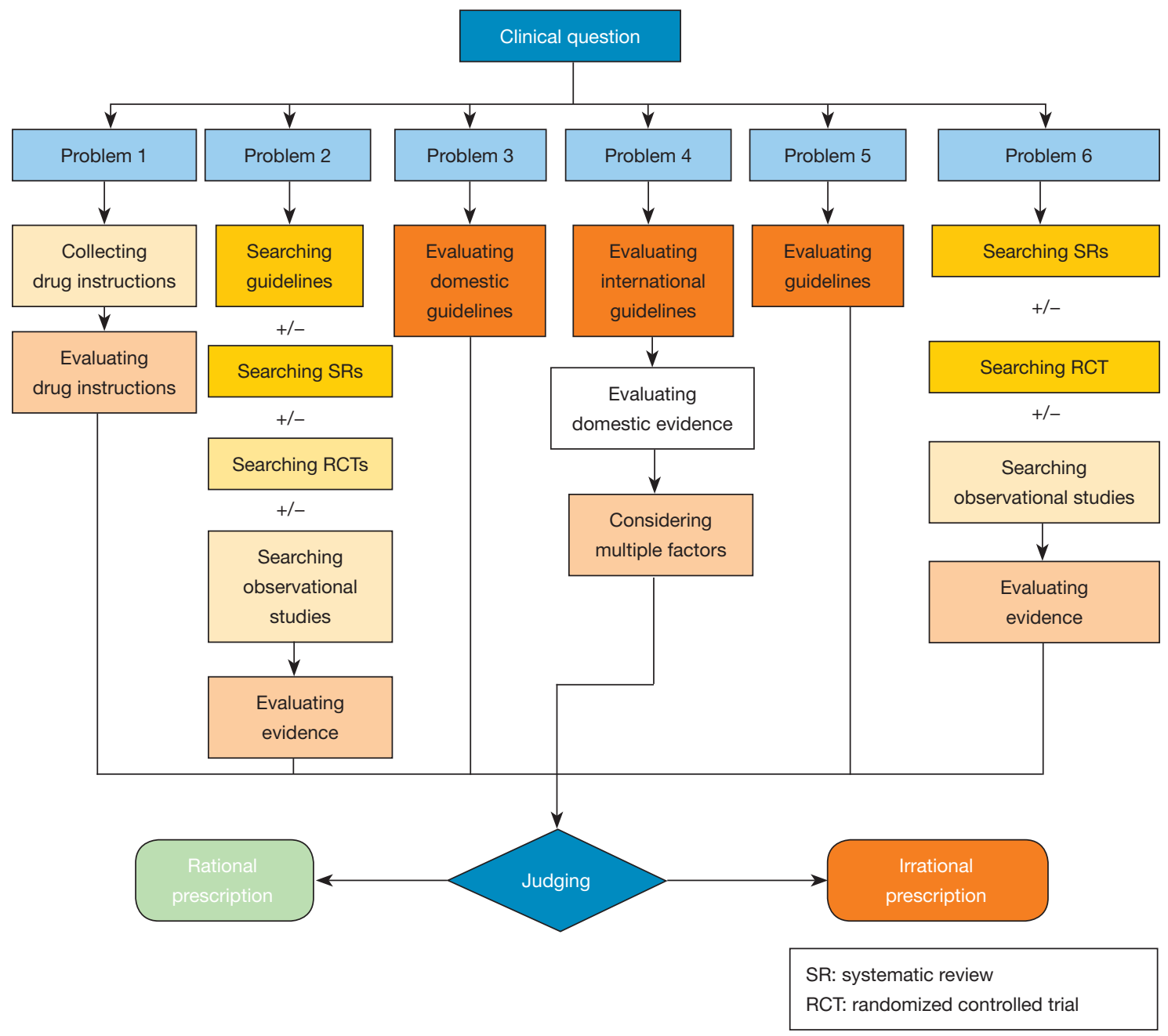

Figure 1 Flow chart for the evaluation of prescription appropriateness.

\section{Acknowledgments}

Funding: None.

\section{Footnote}

Reporting Checklist: The authors have completed the RIGHT reporting checklist. Available at https://dx.doi. org/10.21037/atm-20-7502

Peer Review File: Available at https://dx.doi.org/10.21037/ atm-20-7502

Conflicts of Interest: All authors have completed the ICMJE uniform disclosure form (available at https://dx.doi. org/10.21037/atm-20-7502). The authors have no conflicts of interest to declare.

Ethical Statement: The authors are accountable for all aspects of the work in ensuring that questions related to the accuracy or integrity of any part of the work are appropriately investigated and resolved.

Open Access Statement: This is an Open Access article distributed in accordance with the Creative Commons Attribution-NonCommercial-NoDerivs 4.0 International License (CC BY-NC-ND 4.0), which permits the noncommercial replication and distribution of the article with the strict proviso that no changes or edits are made and the original work is properly cited (including links to both the formal publication through the relevant DOI and the license). See: https://creativecommons.org/licenses/by-nc-nd/4.0/. 


\section{References}

1. Ministry of Health of the PRC. Notice of the ministry of health on printing and distributing the management practices of hospital prescription comment(trial). Available online: http://www.moh.gov.cn/publicfiles/business/ htmlfiles/mohylfwigs/s3582/201003/46163.htm. Accessed June 10, 2020.

2. World Health Organization. WHO Handbook for Guideline Development. Second edition 2014.

3. Ministry of Health of the PRC. Decree of the Ministry of Health of the PRC No. 53: Prescription Management Method. Available online: http://www.gov.cn/flfg/200703/13/content_549406.htm. Accessed June 10, 2020.

4. Haynes RB. Of studies, syntheses, synopses, summaries, and systems: the "5S" evolution of information services for evidence-based healthcare decisions. Evid Based Med 2006;11:162-4.

5. Brouwers MC, Kho ME, Browman GP, et al. AGREE II: advancing guideline development, reporting and evaluation in health care. CMAJ 2010;182:E839-42.

6. Shea BJ, Grimshaw JM, Wells GA, et al. Development of AMSTAR: a measurement tool to assess the methodological quality of systematic reviews. BMC Med

Cite this article as: Bian J, Li Q, Li J, Yang N, Zhang W, Mei D, Zhong M, Miao L, Lu X, Jiang L, Du G, Gao S, Jiang X, Lu J, Yan B, Kong F, Chen Y, Zhen J; represent the guideline expert group from the Pharmaceutical Affairs Commission of the Chinese Hospital Association. Guideline for the evaluation of prescription appropriateness. Ann Transl Med 2021;9(16):1352. doi: 10.21037/atm-20-7502
Res Methodol 2007;7:10.

7. Higgins JP, Altman DG, Gøtzsche PC, et al. The Cochrane Collaboration's tool for assessing risk of bias in randomised trials. BMJ 2011;343:d5928.

8. Whiting PF, Rutjes AW, Westwood ME, et al. QUADAS-2: a revised tool for the quality assessment of diagnostic accuracy studies. Ann Intern Med 2011;155:529-36.

9. Wells GA, Shea BJ, O'Connell D, et al. The NewcastleOttawa Scale (NOS) for assessing the quality of nonrandomized studies in meta-analyses. The Ottawa Hospital Research Institute 2014.

10. Guyatt G, Oxman AD, Akl EA, et al. GRADE guidelines: 1. Introduction-GRADE evidence profiles and summary of findings tables. J Clin Epidemiol 2011;64:383-94.

11. Alonso-Coello P, Schünemann HJ, Moberg J, et al. GRADE Evidence to Decision (EtD) frameworks: a systematic and transparent approach to making well informed healthcare choices. 1: Introduction. BMJ 2016;353:i2016.

12. Becker M, Neugebauer EA, Eikermann M. Partial updating of clinical practice guidelines often makes more sense than full updating: a systematic review on methods and the development of an updating procedure. J Clin Epidemiol 2014;67:33-45. 


\section{Appendix 1}

Diagram 1: Examples of comments for clinical question 1

Example 1: The directions for use of hydroxychloroquine sulfate tablets (Fenle, $100 \mathrm{mg}$ tablet) indicate that it is not a suitable medication for children, while the directions for hydroxychloroquine sulfate tablets (Saineng, $200 \mathrm{mg}$ tablet) indicate that it should not be given to children under 6 years of age. Therefore, is

Comment: If the clinician can provide the drug instructions for hydroxychloroquine sulfate tablets (Saineng, $200 \mathrm{mg}$ tablet) and give a reasonable explanation for their use, the prescription can be considered reasonable. Following this, prescription comment systems should update the drug instructions for related medications.

Example 2: According to the directions, the maximum dose of sodium bicarbonate tablets produced by Beijing Yanjing is $3 \mathrm{~g} /$ day, while that of sodium bicarbonate tablets produced by Tianjin Lisheng is $6 \mathrm{~g} /$ day. For patients with hyperuricemia, the clinician prescribed sodium bicarbonate tablets produced by Beijing Yanjing with a dose of $1.5 \mathrm{~g}$ Tid. Is this prescription reasonable?

Comment: Prescribing sodium bicarbonate tablets (Beijing Yanjing) at $1.5 \mathrm{~g}$ Tid, equivalent to $4.5 \mathrm{~g} /$ day, exceeds the maximum dose recommended by Beijing Yanjing. If the prescriber provides a sodium bicarbonate tablet recommendation (Tianjin Lisheng) and gives reasonable explanation, the prescription may be considered rational. However, the prescribing clinician should be reminded to follow the manufacturer's recommended dosage of a prescribed drug. After this, the prescription comment system should update the instruction for related drugs.

Diagram 2: Examples of comments for clinical question 2

Example 1: Thalidomide has the same pharmacological mechanism for treating the rheumatic immune conditions of ankylosing spondylitis and Behcet's disease. The Guideline for Diagnosis and Treatment of Ankylosing Spondylitis issued by China in 2010 (13) recommend a 50-100 mg/hight dose of thalidomide. The Guideline for Diagnosis and Treatment of Behcet's Disease issued by China in 2011 (14) of 50-100 $\mathrm{m}$, chictis of 50-100 $\mathrm{mg}$, clinicians may prescche prescription reasonable?

the leaflet, thalidomide is used in the treatment of erythema nodosum leprosum in China. The use of thalidomide etment of Behcet's disease and ankylosing spondylitis is off label. There is no evidence supporting a thalidomide dosage of 25 $\mathrm{mg} 3$ times daily for the treatment of ankylosing spondylitis, so these prescriptions may be judged as unreasonable.

Diagram 3: Examples of comments for clinical question 3

Example 1: According to the instructions for use of ferrous succinate tablets, pregnant women should take 2 tablets twice a day for an iron supplement of $120 \mathrm{mg} / \mathrm{day}$. According to the Guideline for Diagnosis and Treatment of Iron Deficiency and Iron-Deficiency Anemia during Pregnancy issued by the Chinese Society of Perinatal Medicine in 2014, pregnant women with iron-deficiency anemia should take iron supplements at 100-200 $\mathrm{mg} /$ day. Clinicians should prescribe 2 tablets of ferrous succinate 3 times a day in order to supplemen pregnant women with $180 \mathrm{mg}$ of iron per day. The dose of this prescription is in line with the guideline but beyond the drug manufacturer's recommended dosage. Is the prescription therefore unreasonable?

Comment: The Guideline for Diagnosis and Treatment of Iron Deficiency and Iron-Deficiency Anemia during Pregnancy issued by the Chinese Society of Perinatal Medicine in 2014 (15) (guideline quality: moderate) recommends that pregnant women with iron-deficiency anemia receive a maximum iron dose of $200 \mathrm{mg} /$ day. A prescribed dose of $180 \mathrm{mg} /$ day of iron supplements is in line with the guideline and can be considered reasonable.

Example 2: Rheumatoid arthritis is not an indication for methotrexate. Is it unreasonable for clinicians to prescribe methotrexate for patients with rheumatoid arthritis as recommended by the Chinese Guideline for the Diagnosis and Treatment of Rheumatoid Arthritis issued by the Chinese Rheumatology Association in 2018?

Comment: The Chinese Guideline for the Diagnosis and Treatment of Rheumatoid Arthritis issued by the Chinese Rheumatology Association in 2018 (16) (guidelines quality: high) recommends methotrexate as the preferred drug for treating rheumatoid arthritis (strongly recommended based on high-quallty evidence). Likewise, recent guidelines issued by several international academic organizations the field of rheumatic disease study, including the American College of Rheumatology (ACR), the European League Against Rheumatism (EULAR), and the Asia Pacific League of Associations for Rheumatology (APLAR) (17-19) (guideline quality: high) all have consistently identified methotrexate as the preferred treatment for patients with rheumatoid arthritis. In summary, the evidence of methotrexate for the treatment of rheumatoid arthritis is sufficient, so the prescription is reasonable.

Example 3: Pregnancy is a contraindication in diazepam's drug label. The Guideline for the Management of Hypertensive Disorders of Pregnancy issued by the Society of Obstetrics and Gynecology from the Chinese Medical Association in 2015 states that diazepam administered orally or by intramuscular injection before bed can help pregnant women relieve the symptoms of mental tension and anxiety, improve sleep, and prevent and control eclampsia. Is it unreasonable for clinicians to prescribe diazepam for pregnant women?

Comment: Diazepam is recommended for use during pregnancy by the Guideline for the Management of Hypertensive Disorders of Pregnancy issued by the Society of Obstetrics and Gynecology from the Chinese Medical Association in 2015 (20) (guideline quality: high) and the Guideline for Epilepsy in Pregnancy issued by the Royal College of Obstetricians and Gynaecologists in 2016 (21). Teratogenic effects of diazepam have not been observed in clinical practice, so the prescription can be judged as reasonable.

Diagram 4: Examples of comments for clinical question 4

Example 1: The approved maximum dose of pantoprazole is $160 \mathrm{mg} /$ day. However, in practice, clinicians may sometimes prescribe pantoprazole at $200-240 \mathrm{mg} /$ day to patients with severe acute gastrointestinal bleeding. Domestic guidelines do not recommend using pantoprazole at $200-240 \mathrm{mg} / \mathrm{day}$ to patients with severe acute gastrointestinal bleeding. Domestic guidelines
high doses of pantoprazole in patients with acute gastrointestinal bleeding, so is this prescription unreasonable?

Comment: The Clinical Guideline for Acute Upper and Lower Gastrointestinal Bleeding issued by the Scottish Intercollegiate Guidelines Network (SIGN) in 2008 (22) (Guideline quality: high) recommends that an intravenous injection of high-dose proton pump inhibitors (infusion of $8 \mathrm{mg}$ hour for 72 hours following an initial intravenous dose of $80 \mathrm{mg}$ ) can be used for pationts receiving ndoscopic treatment (infusion of $8 \mathrm{mg} /$ hour for 72 hours following an initial intravenous dose of $80 \mathrm{mg}$ ) can be used for patients receiving endoscopic treatmen for peptic ulcer bleeding. The guideline issued by the American College of Gastroenterology (ACG) in 2012 (23) (guideline quality: high can reduce the risk of massive bleeding during endoscopic examination and treatment; the ACG further recommends injecting proton $\mathrm{mm}$ inhibitor e after successful hemostasis under endoscopy is reached for patients with active bleeding, exposed blood vessels, or adherent blood clots (infusion of $8 \mathrm{mg} / \mathrm{hour}$ for 72 hours following an initial intravenous dose of $80 \mathrm{mg}$ ). According to a 2007 randomized controlled trial $(n=153)$ from Hong Kong, China (24), for patients with bleeding from endoscopic treatment of peptic ulcer, the use of pantoprazole by intravenous infusion (following an initial intravenous injection of $80 \mathrm{mg}$ of pantoprazole and an $8 \mathrm{mg} / \mathrm{hour}$ continuous infusion lasting for 3 days) or an intravenous injection (following an initial $80 \mathrm{mg}$ intravenous injection of pantoprazole and an infusion of $40 \mathrm{mg}$ every 12 hours for 3 days) can reduce the chance of patient rebleeding (quality of evidence: low). In summary, a high dose ( $>160 \mathrm{mg}$ ) of pantoprazole can be used in patients with acute gastrointestinal bleeding, and there is sufficient evidence to judge that the prescription is reasonable. Example 2: Liraglutide is approved
treat obesity in nondiabetic patients?

Comment: In China, guidelines and textbooks do not provide recommendations or instructions for the treatment of obesity by liraglutide, but it is sometimes used to treat obesity in domestic clinical practice. The Guideline of Pharmacological Management of Obesity issued by the European Endocrine Society in 2015 (25) (guideline quality: high) and the Consensus Statement by the American Association of Clinical Endocrinologist and American College of Endocrinology in 2019 (26) (guideline quality: high) both recommend the use of liraglutide for the treatment of obesity The Food and Drug Administration has approved the use of liraglutide for patients with a BMl above $27 \mathrm{~kg} / \mathrm{m}^{2}$ and at least 1 complication of obesity (such as high blood pressure, type 2 diabetes, hyperlipidemia) or those who have simple obesity with a BMI above $30 \mathrm{~kg} / \mathrm{m}^{2}$. In conclusion, there is sufficient evidence for the treatment of obesity with liraglutide, so the prescription is reasonable.

Diagram 5: Examples of comments for clinical question 5

Example 1: Mycophenolate mofetil is not approved for the treatment of lupus erythematosus in China. The Guideline for the Diagnosis and Treatment of Systemic Lupus Erythematosus (SLE) issued by the Chinese Rheumatology Association in 2010 recommends tha patients with SLE should take mycophenolate mofetil at 0.5-1 g 2 times day. The Guideline for Diagnosis and Treatment of Cutaneous Lupus Erythematosus (CLE) issued by the Chinese Society of Dermatology in 2019 recommends that patients with CLE should tak mycophenolate mofetil, $35 \mathrm{mg} / \mathrm{kg} / \mathrm{day}$, divided into 2 doses. If one patient with lupus erythematosus (LE) weighs $70 \mathrm{~kg}$, the CLE guideline allows for a 2.5g/day dose of mycophenolate mofetil, but the dose is more than that recommended by the SLE guideline. Is it reasonable

Comment: The quality of the SLE guideline from 2010 (27) is low, while the quality of the 2019 CLE guideline (28) is moderate. Prescribing $2.5 \mathrm{~g} / \mathrm{day}$ of mycophenolate mofetil to patients with a body weight of $70 \mathrm{~kg}$ is consistent with the CLE guideline's recommendation mycophenolate mofetil at $35 \mathrm{mg} / \mathrm{kg} / \mathrm{day}$, and as this guideline is of higher quality, the prescription may be judged as reasonable.

Diagram 6: Examples of comments for clinical question 6

Example 1: Tofacitinib is approved in China for the treatment of adult patients with moderate to severe active rheumatoid arthritis for whom methotrexate is ineffective or intolerable. Is it reasonable for a clinician to prescribe oral tofacitinib to patients with severe psoriasis, $5 \mathrm{mg} 3$ times a day?

Comment: There is no recommendation for the treatment of psoriasis by tofacitinib in either domestic or international guidelines. In 2018, a SR from Taiwan, China, (n=2,724) (29) showed that compared to a placebo, tofacitinib significantly reduced the Psoriasis Area and Severity Index score of moderate to severe plaque psoriasis patients and improved the Physician's Global Assessment; however, tofacitinib was also associated with higher cholesterol levels and higher rates of upper respiratory infections. The differences in other adverse reactions between the 2 groups was not statistically significant (quality of evidence: moderate). Thus, there is sufficient evidence for the treatment of severe psoriasis with tofacitinib, so the prescription can be considered reasonable.

Example 2: For some patients with inflammatory arthritis, a specific diagnosis often cannot be established in the weeks and month following the initial onset of symptoms, but medication is required to reduce both patient symptoms and the progression of the disease. There are currently no guidelines for the treatment of inflammatory multijoint disease. Is it unreasonable for clinicians to prescribe hydroxychloroquine sulfate and leflunomide to patients diagnosed with inflammatory multijoint disease?

Comment: There is no recommendation for the use of hydroxychloroquine sulfate combined with leflunomide in the treatment of inflammatory arthritis in either domestic or international guidelines, and no systematic evaluations or original studies about the combination of hydroxychloroquine sulfate and leflunomide in the treatment of inflammatory arthritis have been found. In conclusion, evidence for the treatment of inflammatory multijoint disease with hydroxychloroquine sulfate combined with leflunomide is lacking, so the prescription should be judged as unreasonable. 


\section{References}

13. ACR. Guideline for Diagnosis and Treatment of Ankylosing Spondylitis. Chinese Journal of Rheumatology 2010;14:557-9.

14. ACR. Guideline for Diagnosis and Treatment of Behcet's Disease. Chinese Journal of Rheumatology 2011;15:345-7.

15. Medicine CSoP. Guideline for the diagnosis and treatment of iron deficiency and iron-deficiency anemia during pregnancy. Chinese Journal of Perinatal Medicine 2014;17:451-4.

16. ACR. 2018 Chinese guideline for the diagnosis and treatment of rheumatoid arthritis. Chinese Journal of Internal Medicine 2018;57:242-251.

17. Singh JA SK, Bridges SLJ, et al. 2015 American College of Rheumatology Guideline for the Treatment of Rheumatoid Arthritis. Arthritis Rheumatol 2016;68:1-26.

18. Smolen JS, Landewé RBM, Bijlsma JWJ, et al. EULAR recommendations for the management of rheumatoid arthritis with synthetic and biological disease-modifying antirheumatic drugs: 2016 update. Ann Rheum Dis 2017;76:960-77.

19. Lau CS, Chia F, Harrison A, et al. APLAR rheumatoid arthritis treatment recommendations. Int J Rheum Dis 2015;18:685-713.

20. Association SoOaGoCM. Guideline for the management of hypertensive disorders of pregnancy. Chinese Journal of Obstetrics and Gynecology 2015;50(10):721-728.

21. (RCOG) RCoOaG. Epilepsy in Pregnancy. 2016.

22. Network SIG. Management of acute upper and lower gastrointestinal bleeding: A national clinical guideline. 2008.

23. Laine L, Jensen DM. Management of patients with ulcer bleeding. Am J Gastroenterol 2012;107:345-60; quiz 361.

24. Hung WK, Li VK, Chung CK, et al. Randomized trial comparing pantoprazole infusion, bolus and no treatment on gastric $\mathrm{pH}$ and recurrent bleeding in peptic ulcers. ANZ J Surg 2007;77:677-81.

25. Apovian CM, Aronne LJ, Bessesen DH, et al. Pharmacological management of obesity: an endocrine Society clinical practice guideline. J Clin Endocrinol Metab 2015;100:342-62. Correction in J Clin Endocrinol Metab 2015;100:2135-6.

26. Garber AJ, Abrahamson MJ, Barzilay JI, et al. Consensus Statement by the American Association Of Clinical Endocrinologists and American College of Endocrinology on the Comprehensive Type 2 Diabetes Management Algorithm - 2019 Executive Summary. Endocr Pract 2018;24:91-120.

27. ACR. Guideline for the diagnosis and treatment of systemic lupus erythematosus. Chinese Journal of Rheumatology 2010;14:342-6.

28. Dermatology CfLERoCSo. Guideline for diagnosis and treatment of cutaneous lupus erythematosus (2019). Chinese Journal of Dermatology 2019;52:149-55.

29. Kuo CM, Tung TH, Wang SH, et al. Efficacy and safety of tofacitinib for moderate-to-severe plaque psoriasis: a systematic review and meta-analysis of randomized controlled trials. J Eur Acad Dermatol Venereol 2018;32:355-62. 